\title{
Many-body localization transition in large quantum spin chains: The mobility edge
}

\author{
Titas Chanda $\oplus^{1, *}$ Piotr Sierant, ${ }^{1, \dagger}$ and Jakub Zakrzewski $\oplus^{1,2, \ddagger}$ \\ ${ }^{1}$ Instytut Fizyki Teoretycznej, Uniwersytet Jagielloński, Łojasiewicza 11, 30-348 Kraków, Poland \\ ${ }^{2}$ Mark Kac Complex Systems Research Center, Uniwersytet Jagielloński, Kraków, Poland
}

(Received 9 June 2020; revised 28 July 2020; accepted 5 August 2020; published 21 August 2020)

\begin{abstract}
Thermalization of random-field Heisenberg spin chain is probed by time evolution of density correlation functions. Studying the impacts of average energies of initial product states on dynamics of the system, we provide arguments in favor of the existence of a mobility edge in the large system-size limit.
\end{abstract}

DOI: 10.1103/PhysRevResearch.2.032045

Introduction. Many-body localization (MBL) [1,2] is a robust mechanism that prevents quantum many-body systems from reaching of thermal equilibrium [3-5]. The phenomenon, originating from an interplay of interactions and disorder [6-8], has been studied numerically in various models: spin chains [9-12] that map onto spinless fermionic chains, and spinful fermions [13-16] or bosons [17-19]. Despite those efforts, a complete understanding of the transition between ergodic and MBL phases is still lacking. While the recent works [20-24] suggest a Kosterlitz-Thouless scaling at the MBL transition, it became clear that the exact diagonalization studies are subject to strong finite-size effects [25-27] that prevent one from reaching unambiguous conclusions about the thermodynamic limit [28,29].

Alternatively, time evolution of large [30] (or even infinite [31]) disordered many-body systems can be simulated with tensor network algorithms. Reaching the large timescales necessary to assess thermalization properties [32] is challenging, especially in the vicinity of the transition to the ergodic phase. Nevertheless, such an approach allows one to obtain estimates for critical disorder strength for large system sizes [30,32], in quasiperiodic systems [33], or even beyond one spatial dimension $[34,35]$. An advantage of such an approach is that it directly mimics experimental observations of MBL [36-43].

Typically, the transition between ergodic and MBL phases is induced by tuning the disorder strength. Then the natural extension is whether one can envision a different control parameter. In this work, we consider energy as such a parameter. This immediately translates to a problem of the existence of many-body mobility edges, i.e., energies that separate localized and extended states [2]. The many-body mobility edges observed in early exact diagonalization studies of small

\footnotetext{
*titas.chanda@uj.edu.pl

†piotr.sierant@uj.edu.pl

*jakub.zakrzewski@uj.edu.pl
}

Published by the American Physical Society under the terms of the Creative Commons Attribution 4.0 International license. Further distribution of this work must maintain attribution to the author(s) and the published article's title, journal citation, and DOI. systems $[12,44,45]$ were argued to be indistinguishable from finite-size effects in Ref. [46]. The argument of Ref. [46] is that local fluctuations in a system with a putative manybody mobility edge can serve as mobile bubbles, inducing a global delocalization, and hence no many-body mobility edge can exist. The existence of mobility edges is one of the fundamental problems of MBL; it leads to questions about the phenomenology of systems with many-body mobility edge (since the description in terms of local integrals of motion [47-53] does not apply in such a scenario), or to the anomalous dynamics for a nonstationary initial state due to energy fluctuations [54,55].

The aim of our work is to study many-body mobility edges at much larger system sizes than those available to exact diagonalization in an attempt to verify conclusions of Ref. [46]. To that end, we employ the Chebyshev polynomial expansion of the evolution operator [56-58] and the timedependent variational principle (TDVP) applied to matrix product states (MPS) [59-62] to simulate time dynamics of a random-field Heisenberg spin chain. Our approach is, in spirit, similar to that of Refs. [63,64] (and used for bosons in Ref. [65]). However, instead of considering an injection of a controllable amount of energy into the ground state of the system, we consider time evolution of initial product states with specified average energies, exactly what was done recently in a spin quantum simulator [66]. Probing time decay of density correlation functions allows us to estimate the critical disorder strength as a function of energy of the initial state. Studying systems of size up to $L=100$, we perform a finite-size scaling of our results, which provides arguments in favor of the existence of mobility edges even in large systems.

The model and methods. We consider a one-dimensional (1D) random-field Heisenberg $(X X Z)$ spin chain with the Hamiltonian given by

$$
H=J \sum_{i=1}^{L-1}\left(S_{i}^{x} S_{i+1}^{x}+S_{i}^{y} S_{i+1}^{y}+S_{i}^{z} S_{i+1}^{z}\right)+\sum_{i=1}^{L} h_{i} S_{i}^{z},
$$

where $S_{i}^{\alpha}, \alpha=x, y, z$, are spin-1/2 matrices, $J=1$ is fixed to be the unit of energy, and $h_{i} \in[-W, W]$ are independent, uniformly distributed random variables. In this work, we consider open boundary conditions in the Hamiltonian (1). The 


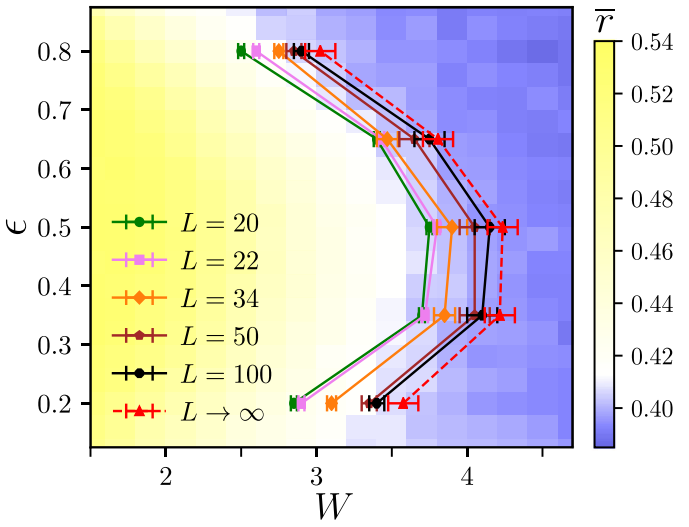

FIG. 1. Phase diagram of random-field Heisenberg spin chain, disorder strength $W$ on horizontal axis, rescaled energy $\epsilon$ on vertical axis. Background shows color-coded value of average gap ratio $\bar{r}$ for system size $L=16$. Solid lines show the position of boundary between ergodic and MBL phases obtained in study of decay of density correlations in systems of size $L=20,22,26,34,50,100$, and dashed line shows results of extrapolation of the results to $L \rightarrow \infty$.

random-field Heisenberg spin chain has been widely studied in the MBL context [12,31,67-73], which has made it the de facto standard model of MBL studies.

The transition between ergodic and MBL phases is reflected in change of statistical properties of energy levels of the system. A common approach is to consider the gap ratio $r_{i}=\frac{\min \left\{E_{i+2}-E_{i+1}, E_{i+1}-E_{i}\right\}}{\max \left\{E_{i+2}-E_{i+1}, E_{i+1}-E_{i}\right\}}$, where $E_{i}$ are the energy eigenvalues of the system. Averaging the gap ratio over part of the spectrum of the system and over disorder realizations, one obtains an average gap ratio $\bar{r}$, which differentiates between level statistics of ergodic system [10,74], well described by Gaussian orthogonal ensemble of random matrices, for which $\bar{r} \approx 0.53$ and between Poissonian statistics of eigenvalues in MBL phase (for which $\bar{r} \approx 0.39$ ). The later arises due to emergent integrability resulting from the presence of local integrals of motion [47-53].

To reveal the dependence of an ergodic-MBL transition on energy, the gap ratios $r_{i}$ are averaged over only a certain number of eigenvalues with energies close to a rescaled energy $\epsilon=$ $\left(E-E_{\min }\right) /\left(E_{\max }-E_{\min }\right)$, where $E_{\min }\left(E_{\max }\right)$ is the energy of the ground (highest excited) state. Such a calculation of average gap ratio (supported with results for other probes of localization) for a random-field Heisenberg spin chain reveals that the ergodic region has shape of a characteristic lobe on the phase diagram in variables of the rescaled energy $\epsilon$ and disorder strength $W$ [12]. The average gap ratio, obtained in exact diagonalization of random-field Heisenberg spin chain of size $L=16$, is plotted as a function of $\epsilon$ and $W$ in the background of Fig. 1.

To probe the transition between ergodic and MBL phases with time evolution, we propose the following protocol. We consider an initial state $|\psi\rangle=\left|\sigma_{1}, \ldots, \sigma_{L}\right\rangle$, where $\sigma_{i}=\uparrow, \downarrow$ are chosen randomly with constraint that the average rescaled energy $\epsilon_{\psi}=\left(\langle\psi|H| \psi\rangle-E_{\min }\right) /\left(E_{\max }-E_{\min }\right)$ of this state lies within the range $[\epsilon-\delta \epsilon, \epsilon+\delta \epsilon]$ corresponding to a given rescaled energy $\epsilon$, where $\delta \epsilon$ is a small tolerance (we take
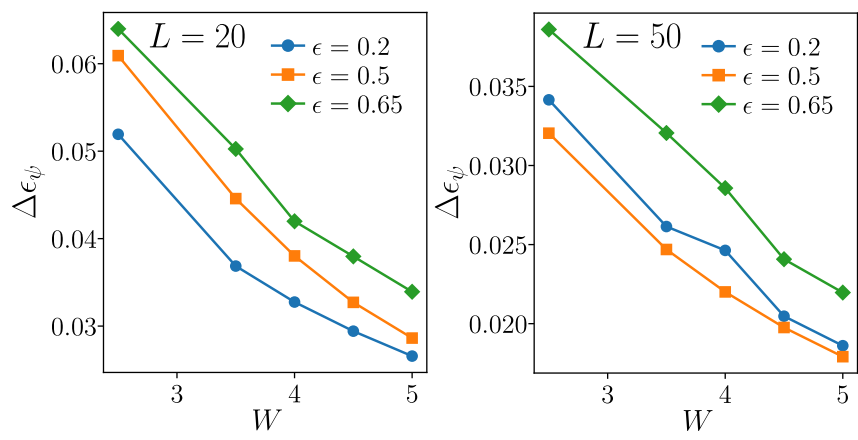

FIG. 2. Disorder averaged standard deviation $\Delta \epsilon_{\psi}$ of rescaled energy of the initial states as a function of disorder strength $W$ for three exemplary rescaled energies $\epsilon$. Left, system size $L=20$; right, system size: $L=50$.

$\delta \epsilon=0.01)$. To calculate $\epsilon_{\psi}$ for $L \leqslant 26$, we find $E_{\max }, E_{\min }$ with the standard Lanczos algorithm [75]. For larger system sizes, $E_{\min }$ and $E_{\max }$ are calculated using the density matrix renormalization group (DMRG) algorithm [76-80] (see Ref. [81] for details).

Subsequently, we calculate time evolved state $|\psi(t)\rangle=$ $e^{-i H t}|\psi\rangle$ with the standard Chebyshev expansion of the evolution operator [58] for $L \leqslant 26$. For larger system sizes, we use the recently developed TDVP algorithm [59-62]. Technically, we follow Refs. [32,82] and employ a hybrid of two-site and one-site versions of TDVP [62,83] (see Ref. [81] for details).

Our quantity of interest is the density correlation function

$$
C(t)=D \sum_{i=1+l_{0}}^{L-l_{0}}\left\langle\psi(t)\left|S_{i}^{z}\right| \psi(t)\right\rangle\left\langle\psi\left|S_{i}^{z}\right| \psi\right\rangle,
$$

where the constant $D$ assures that $C(0)=1$ and $l_{0}>0$ diminish the influence of boundaries (in our calculations, we take $l_{0}=2$ ). The standard deviation of the rescaled energy

$$
\Delta \epsilon_{\psi}=\left\{\left\langle\psi\left|\left[\left(H-E_{\min }\right) /\left(E_{\max }-E_{\min }\right)-\epsilon_{\psi}\right]^{2}\right| \psi\right\rangle\right\}^{1 / 2}
$$

is smaller than 0.1 for disorder strengths that we consider in this work as shown in Fig. 2. Those relatively small fluctuations of energy suggest that the properties of eigenstates at the rescaled energy $\epsilon$ can be well probed by time evolution of the state $|\psi\rangle$ and reflected, in particular, by the density correlation function $C(t)$.

Quench dynamics: Dependence on disorder strength. Figure 3 shows the density correlation functions $C(t)$ obtained for the random-field Heisenberg spin chain of a fixed size $L=20$, for rescaled energy $\epsilon=0.5$ of the initial state. The correlation function decreases in time, with some oscillations superimposed [84]. For small disorder strength, e.g., $W=2.8$, the eigenstate thermalization hypothesis $[5,85]$ is valid for the system, and in the long time limit the correlation function vanishes $C(t) \stackrel{t \rightarrow \infty}{\rightarrow} 0$ as system loses the memory of the initial state. In contrast, for large disorder strengths, e.g., $W=5$, a nonzero stationary value of the correlation function $C(t) \stackrel{t \rightarrow \infty}{\rightarrow}$ $c_{0}>0$ is admitted, showing that the system is nonergodic. The first experimental signatures of MBL were obtained in study of time evolution of imbalance [36], quantity analogous 


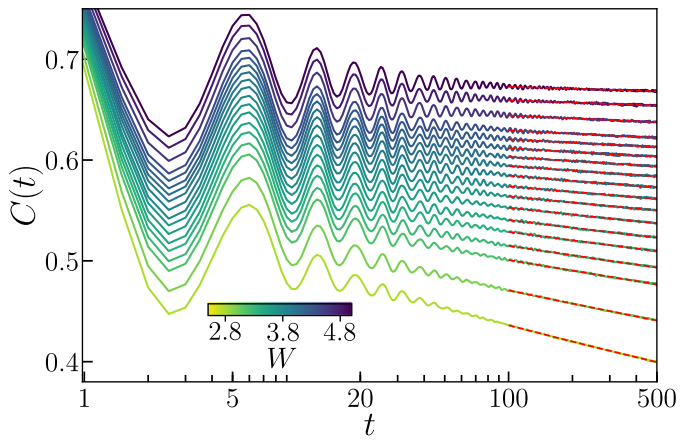

FIG. 3. Quench dynamics in disordered $X X Z$ spin chain. Density correlation function $C(t)$ for system size $L=20$ and various disorder strengths $W=2.8, \ldots, 5$ (color coded) averaged over 10000 disorder realizations, rescaled energy of the initial state $\epsilon=0.5$, and powerlaw fits $C(t) \propto t^{-\beta}$ for $t \in[100,500]$ are denoted by the dashed lines.

to the density correlation function-for quantitative comparison of the two quantities, see Ref. [81].

At large times $(t>100)$, the decay of the correlation function is well described by a power law, $C(t) \propto t^{-\beta}$. Griffiths rare regions are one possible explanation of this behavior [86]. However, it was shown experimentally and numerically that time dynamics in quasiperiodic potentials, where Griffiths regions are necessarily absent, have analogous features $[70,87,88]$. Regardless of the origin of the power law decay of the correlation function, the disorder strength dependence of the exponent $\beta$ can be used to locate the onset of ergodicity breaking in the system.

The exponent $\beta$ governing the decay of the density correlation function is shown in Fig. 4(a). Let us first concentrate on the results in the middle of the spectrum $(\epsilon=0.5)$. In the considered interval of disorder strength $W$, the exponent decreases exponentially with $W$ with a good approximation $\beta \propto$ $e^{-W / \Omega}$. The large number of disorder realizations $(10000)$ used in calculation of $C(t)$ allows us to see that even at the large disorder strength $W=5$ the exponent $\beta=4.1(4) \times$

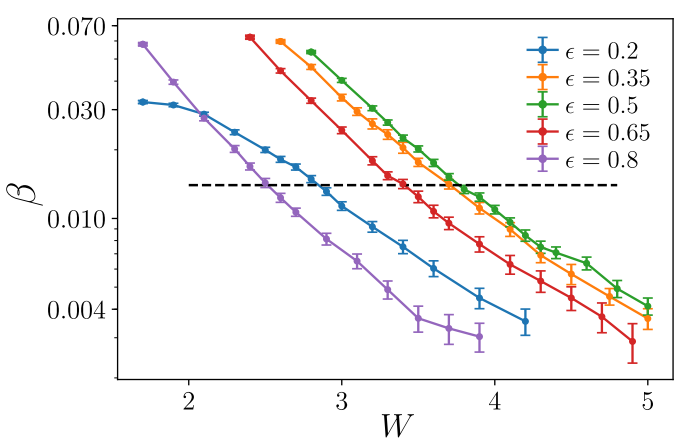

FIG. 4. The exponent $\beta$, obtained in fitting the density correlation function $C(t)$ with an algebraic decay $a_{0} t^{-\beta}$ in interval $t \in$ $[100,500]$, is plotted as function of disorder strength $W$. The error bars represent the $1 \sigma$ errors of the fitting obtained from statistical resampling of disorder realizations. The system size is $L=20$, and results for various rescaled energies $\epsilon$ of the initial state are shown. The dashed line shows the cutoff exponent $\beta_{0}=0.014$.
$10^{-3}$ is nonvanishing. If the power-law decay $C(t)=a_{0} t^{-\beta}$ prevailed for $t \rightarrow \infty$, the density correlation function would vanish in the long-time limit and the system would be ergodic. This, however, does not happen for $L=20$, as after the socalled Heisenberg time $t_{H}$ discreteness of spectrum manifests itself in saturation of the correlation functions [89-92], so that one would observe $C(t) \stackrel{t \rightarrow \infty}{\rightarrow} c_{0}>0$ for $W=5$ and $L=$ 20. The Heisenberg time $t_{H}$ increases exponentially with the system size $L$. This illustrates a difficulty in locating the MBL transition using time dynamics of large systems on timescales of few hundred $J^{-1}$ accessible to tensor network methods (or to current experiments with, e.g., ultracold atoms): One cannot predict whether a slow decay of correlation functions governed by an exponent $\beta \ll 1$ observed, for example, $t \in$ $[100,500]$, will eventually lead to $C(t) \stackrel{t \rightarrow \infty}{\rightarrow} 0$ or not.

To resolve the difficulties, the work of Ref. [30] assumes that the value of the exponent $\beta$ must be vanishing within error bars to be compatible with saturation of correlation functions in the long time limit. The drawback of this criterion is that the error bar of $\beta$ depends on the number of disorder realizations used in calculation of the correlation function. Therefore, we introduce a cutoff $\beta_{0}$ : disorder strength $W_{C}(L)$ for which $\beta=\beta_{0}$ is regarded as disorder strength for transition to MBL phase at system size $L$. Exact diagonalization results show that (i) collapse of data for $L \leqslant 22$ gives a critical disorder strength $W_{C} \approx 3.7$; (ii) the similar values $W_{C} \approx 3.8$ or $W_{C} \approx$ 4.2 are obtained in asymmetric scaling on the two sides of the transition; and (iii) the breakdown of the volume-law scaling of entanglement entropy gives an estimate $W_{C}=3.75$ at system size $L=20$ [29]. The obtained results for $\beta$ at $L=20$ and $\epsilon=0.5$ show that the cutoff value $\beta_{0}=0.014$ is consistent with the above estimates for the critical disorder strength obtained from exact diagonalizations; see Fig. 4. The assumed cutoff value $\beta_{0}$ is nearly independent of the target energy and system size (for further details, see Ref. [81]). Consequently, throughout this work, we use $\beta_{0}=0.014$ as a threshold value which separates ergodic and MBL regimes for all system sizes and energies of the initial state we consider.

The values of $\beta$ presented in Fig. 4(a) show that the increase of disorder strength $W$ slows down the dynamics more severely for rescaled energies of initial state different than $\epsilon=0.5$. Notably, the exponent $\beta$ decreases exponentially with $W: \beta \propto e^{-W / \Omega}$ (where $\Omega$ is a constant) in a wide regime of disorder strengths. This resembles the scaling of Thouless time $t_{T h} \propto e^{-W / W_{0}}$ observed in exact diagonalization data in Ref. [25].

Quench dynamics: Dependence on system size. Density correlation function $C(t)$ for larger system sizes are shown for two exemplary pairs of disorder strength $W$ and initial rescaled energy $\epsilon$ in Fig. 5. The decay of $C(t)$ at large times is well fitted by an algebraic dependence $C(t) \propto t^{-\beta}$. The exponents $\beta$ obtained in the fitting of power-law decay to $C(t)$ are shown for two exemplary values of the rescaled energy $\epsilon$ of the initial states in Fig. 6. For a given disorder strength $W$, we observe a clear increase of $\beta$ with increasing system size. Interestingly, the shift is, to a good approximation, uniform for all disorder strengths so that the exponential decrease $\beta \propto$ $e^{-W / \Omega}$ (at sufficiently large $W$ ) is observed for all considered system sizes. Let us mention here that we consider 400 

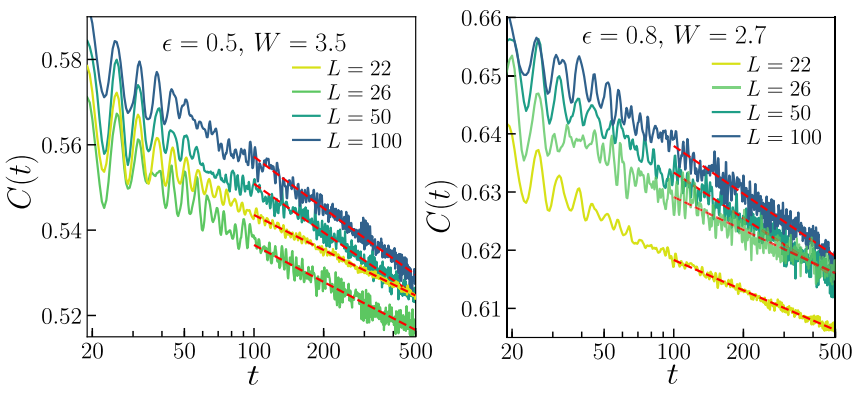

FIG. 5. Time evolution of density correlation function $C(t)$ for rescaled energy of initial state $\epsilon=0.5(\epsilon=0.8)$ and disorder strength $W=3.5(W=2.7)$ on the right (left). The system size $L$ varies from 22 to 100 . The dashed lines denote power-law fits $C(t)=a_{0} t^{-\beta}$ in the $t \in[100,500]$ interval.

realizations of disorder for $L=34,50$, and 200 realizations for $L=100$ for each values of $\epsilon$ and $W$. For small system sizes $(L=20,22,26)$, we consider between 10000 and 500 disorder realizations.

We obtain estimates for disorder strength $W_{C}(L)$ for transition to MBL phase by finding the crossings of $\beta(W)$ curve for given system size $L$ with the $\beta=\beta_{0}$ line. Results of this procedure are shown in Fig. 7(a). The disorder strength $W_{C}(L)$ depends, within the estimated error bars, linearly on the inverse of the system size $L$ with clear growth of $W_{C}(L)$ as the system size increases. On one hand, this trend allows us, by means of a linear fit $W_{C}(1 / L)=A / L+W_{C}(\infty)$, to extrapolate the results to $L \rightarrow \infty$ and to obtain the estimate of critical disorder strength $W_{C}(\infty)$ for transition to MBL phase.

On the other hand, we observe that the slopes $A$ are similar for all of the considered rescaled energies of the initial state. Thus, the shape of the boundary between ergodic and MBL regimes observed for $L=20$ does not change considerably when the system size is increased. This is visible in Fig. 1. The points for various system sizes $L$ are precisely the values of $W_{C}(L)$ obtained from the condition $\beta=\beta_{0}$. The characteristic shape of the lobe does not change when the system sizes increases from $L=20$ to $L=100$ and is preserved even after
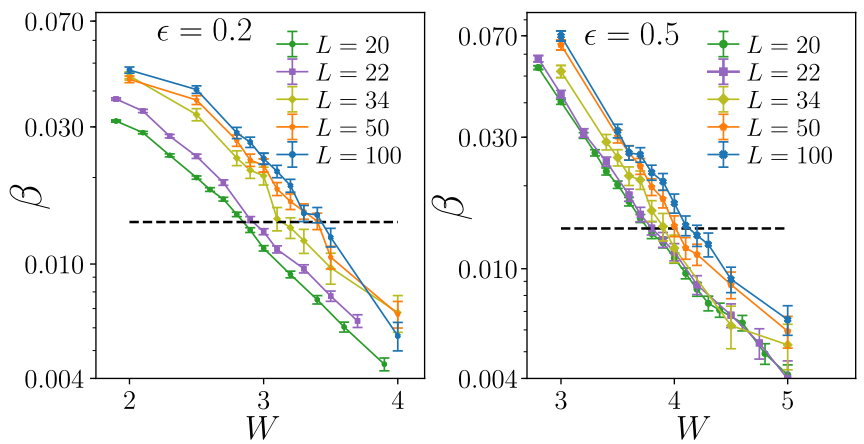

FIG. 6. The exponent $\beta$ obtained in fitting the density correlation function $C(t)$ with an algebraic decay $a_{0} t^{-\beta}$ in interval $t \in$ $[100,500]$ for the rescaled energy $\epsilon=0.2(\epsilon=0.5)$ of the initial state shown in the left (right) panel. Data shown for system sizes $L=20,22,34,50,100$. The error bars show $1 \sigma$ errors of $\beta$ obtained in resampling of disorder realizations. The dashed lines show the cutoff exponent $\beta_{0}$.
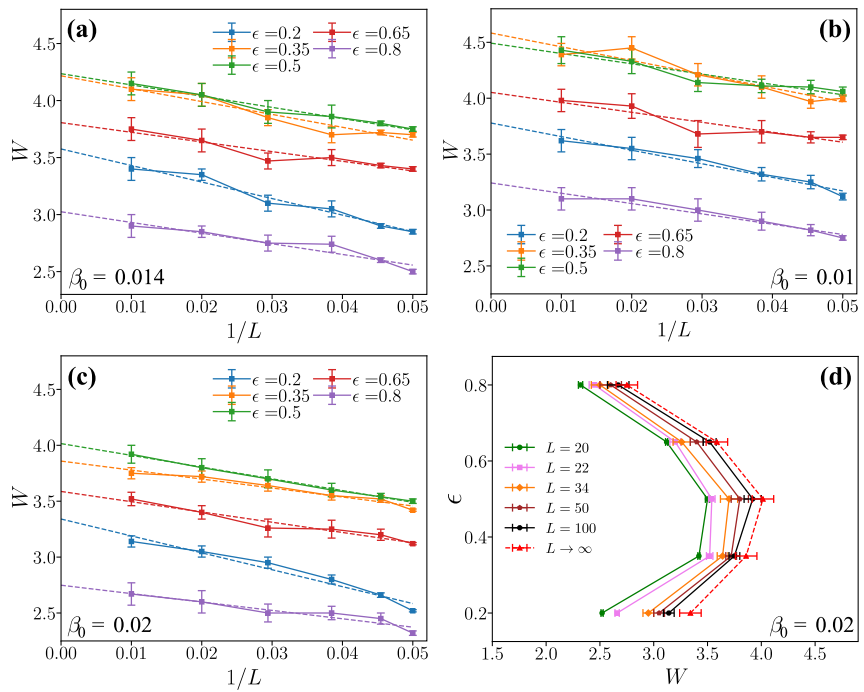

FIG. 7. (a) Disorder strength $W_{C}(L)$ for which decay of correlation function is governed by power law with $\beta=\beta_{0}$ plotted as function of $1 / L$ where $L$ is the system size. Results shown for various rescaled energies $\epsilon$ of initial state. Available data are fitted with linear functions $W_{C}(1 / L)=a / L+W_{C}(\infty)$ which allows extrapolation to $L \rightarrow \infty$. [(b), (c)] Same as in panel (a), but with two different choices of cutoff $\beta_{0}$ (0.01 and 0.02 respectively). (d) The shape of the edge between localized and delocalized regions in $(\epsilon, W)$ plane for different system-sizes $L=20,20,34,50,100$ obtained with threshold $\beta_{0}=0.02$. Dashed line shows the extrapolation of the results for $L \rightarrow \infty$.

the extrapolation to $L \rightarrow \infty$. Therefore, there exists a certain range of disorder strengths such that the states for $\epsilon<\epsilon_{M E}^{L}$ are localized, states for $\epsilon_{M E}^{L}<\epsilon<\epsilon_{M E}^{U}$ are extended, and states for $\epsilon>\epsilon_{M E}^{U}$ are again localized. Thus, our results indicate that the system indeed possesses a many-body mobility edge in the thermodynamic limit.

So far, the results have been reported with the threshold value $\beta_{0}=0.014$. However, the qualitative results and the conclusion about the existence of mobility edge in large systems remain unaltered for different choices of $\beta_{0}$, which we show in Figs. 7(b) and 7(c) by considering $\beta_{0}=0.01$ and 0.02 respectively. However, very small choice of $\beta_{0}$ (e.g., 0.01) results in larger error bars, which points toward the difficulty of obtaining the saturation of the correlation function within finite interval of time with finite number of disorder realizations. Figure 7(d) shows the shapes of the boundary between MBL and delocalized obtained for $\beta_{0}=$ 0.02 at different system sizes, which remain qualitatively the same as those for $\beta_{0}=0.014$.

Discussion and outlook. Chebyshev polynomial expansion of the time evolution operator and the TDVP method applied to MPS allowed us to study the problem of energy dependence of the transition between ergodic and MBL phases in large disordered quantum spin chains. Introducing a cutoff value of exponent $\beta$ of power-law decay in time of density correlation function, we were able to probe the transition for different rescaled energies of the initial state. For small system-sizes (e.g., $L=20$ ), our approach gives results consistent with exact diagonalization. Importantly, our method allows us to 
consider much larger system sizes $(L=100)$ for which it predicts an existence of a mobility edge.

The disorder strength $W_{C}(L)$ is a lower bound on the transition to MBL phase: The residual decay of density correlations with exponent $\beta_{0}$ is insufficient to restore the uniform density profile for system size $L=20$, but it is possible that it leads to an eventual decay of correlation function for larger system sizes.

The protocol we considered is, in principle, experimentally realizable. Our results can be verified experimentally if the setup of Ref. [66] was scaled to larger system sizes. Manybody mobility edge arises also in disordered Bose-Hubbard models [93]. It can be probed by a quench protocol analogous to the one considered in this work. Since the bosonic models allow for occupations in each site larger than unity, density wave-like states that are easier to obtain in an experiment with ultracold atoms can be use to probe the many-body mobility edge $[65,93]$. Recently, we learned about a study [94] where many-body mobility edges with respect to particle numbers were shown to exist in a correlated hopping model of hardcore bosons.

Acknowledgments. Support of the Polish National Science Centre via grants Unisono 2017/25/Z/ST2/03029 (T.C.) (under QTFLAG Quantera Collaboration), Opus 2015/19/B/ST2/01028 (P.S.), and Opus 2019/35/ B/ST2/00034 (J.Z.) is acknowledged. P.S. thanks the Polish National Science Centre for an additional support via Etiuda programme 2018/28/T/ST2/00401 as well as the Foundation for Polish Science (FNP) through scholarship START. The partial support by PL-Grid Infrastructure is also acknowledged. The MPS-based techniques have been implemented using ITensor library v2 [95].
[1] I. V. Gornyi, A. D. Mirlin, and D. G. Polyakov, Phys. Rev. Lett. 95, 206603 (2005).

[2] D. Basko, I. Aleiner, and B. Altschuler, Ann. Phys. (NY) 321, 1126 (2006).

[3] J. M. Deutsch, Phys. Rev. A 43, 2046 (1991).

[4] M. Srednicki, Phys. Rev. E 50, 888 (1994).

[5] M. Rigol, V. Dunjko, and M. Olshanii, Nature (London) 452, 854 (2008).

[6] R. Nandkishore and D. A. Huse, Ann. Rev. Cond. Mat. Phys. 6, 15 (2015).

[7] F. Alet and N. Laflorencie, C. R. Phys. 19, 498 (2018).

[8] D. A. Abanin, E. Altman, I. Bloch, and M. Serbyn, Rev. Mod. Phys. 91, 021001 (2019).

[9] L. F. Santos, G. Rigolin, and C. O. Escobar, Phys. Rev. A 69 , 042304 (2004).

[10] V. Oganesyan and D. A. Huse, Phys. Rev. B 75, 155111 (2007).

[11] A. Pal and D. A. Huse, Phys. Rev. B 82, 174411 (2010).

[12] D. J. Luitz, N. Laflorencie, and F. Alet, Phys. Rev. B 91, 081103(R) (2015).

[13] R. Mondaini and M. Rigol, Phys. Rev. A 92, 041601(R) (2015).

[14] P. Prelovšek, O. S. Barišić, and M. Žnidarič, Phys. Rev. B 94, 241104(R) (2016).

[15] J. Zakrzewski and D. Delande, Phys. Rev. B 98, 014203 (2018).

[16] M. Kozarzewski, P. Prelovšek, and M. Mierzejewski, Phys. Rev. Lett. 120, 246602 (2018).

[17] P. Sierant, D. Delande, and J. Zakrzewski, Phys. Rev. A 95, 021601(R) (2017).

[18] T. Orell, A. A. Michailidis, M. Serbyn, and M. Silveri, Phys. Rev. B 100, 134504 (2019).

[19] M. Hopjan and F. Heidrich-Meisner, Phys. Rev. A 101, 063617 (2020).

[20] A. Goremykina, R. Vasseur, and M. Serbyn, Phys. Rev. Lett. 122, 040601 (2019)

[21] A. Morningstar and D. A. Huse, Phys. Rev. B 99, 224205 (2019).

[22] P. T. Dumitrescu, A. Goremykina, S. A. Parameswaran, M. Serbyn, and R. Vasseur, Phys. Rev. B 99, 094205 (2019).

[23] N. Laflorencie, G. Lemarié, and N. Macé, arXiv:2004.02861.

[24] J. Šuntajs, J. Bonča, T. Prosen, and L. Vidmar, Phys. Rev. B 102, 064207 (2020).
[25] J. Šuntajs, J. Bonča, T. Prosen, and L. Vidmar, arXiv:1905.06345.

[26] P. Sierant, D. Delande, and J. Zakrzewski, Phys. Rev. Lett. 124, 186601 (2020).

[27] D. A. Abanin, J. H. Bardarson, G. D. Tomasi, S. Gopalakrishnan, V. Khemani, S. A. Parameswaran, F. Pollmann, A. C. Potter, M. Serbyn, and R. Vasseur, arXiv:1911.04501.

[28] R. K. Panda, A. Scardicchio, M. Schulz, S. R. Taylor, and M. Žnidarič, EPL 128, 67003 (2020).

[29] P. Sierant, M. Lewenstein, and J. Zakrzewski, arXiv:2005.09534.

[30] E. V. H. Doggen, F. Schindler, K. S. Tikhonov, A. D. Mirlin, T. Neupert, D. G. Polyakov, and I. V. Gornyi, Phys. Rev. B 98, 174202 (2018).

[31] T. Enss, F. Andraschko, and J. Sirker, Phys. Rev. B 95, 045121 (2017).

[32] T. Chanda, P. Sierant, and J. Zakrzewski, Phys. Rev. B 101, 035148 (2020).

[33] E. V. H. Doggen and A. D. Mirlin, Phys. Rev. B 100, 104203 (2019).

[34] C. Hubig and J. I. Cirac, SciPost Phys. 6, 31 (2019).

[35] E. V. H. Doggen, I. V. Gornyi, A. D. Mirlin, and D. G. Polyakov, arXiv:2002.07635.

[36] M. Schreiber, S. S. Hodgman, P. Bordia, H. P. Lüschen, M. H. Fischer, R. Vosk, E. Altman, U. Schneider, and I. Bloch, Science 349, 842 (2015).

[37] P. Bordia, H. Lüschen, U. Schneider, M. Knap, and I. Bloch, Nat. Phys. 13, 460 (2017).

[38] T. Kohlert, S. Scherg, X. Li, H. P. Lüschen, S. Das Sarma, I. Bloch, and M. Aidelsburger, Phys. Rev. Lett. 122, 170403 (2019).

[39] A. Lukin, M. Rispoli, R. Schittko, M. E. Tai, A. M. Kaufman, S. Choi, V. Khemani, J. Léonard, and M. Greiner, Science 364, 256 (2019).

[40] M. Rispoli, A. Lukin, R. Schittko, S. Kim, M. E. Tai, J. Léonard, and M. Greiner, Nature (London) 573, 385 (2019).

[41] J.-y. Choi, S. Hild, J. Zeiher, P. Schauß, A. Rubio-Abadal, T. Yefsah, V. Khemani, D. A. Huse, I. Bloch, and C. Gross, Science 352, 1547 (2016). 
[42] J. Smith, A. Lee, P. Richerme, B. Neyenhuis, P. W. Hess, P. Hauke, M. Heyl, D. A. Huse, and C. Monroe, Nat. Phys. 12, 907 (2016).

[43] K. Xu, J.-J. Chen, Y. Zeng, Y.-R. Zhang, C. Song, W. Liu, Q. Guo, P. Zhang, D. Xu, H. Deng, K. Huang, H. Wang, X. Zhu, D. Zheng, and H. Fan, Phys. Rev. Lett. 120, 050507 (2018).

[44] J. A. Kjäll, J. H. Bardarson, and F. Pollmann, Phys. Rev. Lett. 113, 107204 (2014).

[45] I. Mondragon-Shem, A. Pal, T. L. Hughes, and C. R. Laumann, Phys. Rev. B 92, 064203 (2015).

[46] W. De Roeck, F. Huveneers, M. Müller, and M. Schiulaz, Phys. Rev. B 93, 014203 (2016).

[47] M. Serbyn, Z. Papić, and D. A. Abanin, Phys. Rev. Lett. 111, 127201 (2013).

[48] D. A. Huse, R. Nandkishore, and V. Oganesyan, Phys. Rev. B 90, 174202 (2014).

[49] V. Ros, M. Mueller, and A. Scardicchio, Nucl. Phys. B 891, 420 (2015).

[50] J. Z. Imbrie, Phys. Rev. Lett. 117, 027201 (2016).

[51] T. B. Wahl, A. Pal, and S. H. Simon, Phys. Rev. X 7, 021018 (2017).

[52] M. Mierzejewski, M. Kozarzewski, and P. Prelovšek, Phys. Rev. B 97, 064204 (2018).

[53] S. J. Thomson and M. Schiró, Phys. Rev. B 97, 060201(R) (2018).

[54] D. J. Luitz and Y. B. Lev, Ann. Phys. 529, 1600350 (2017).

[55] D. J. Luitz and Y. Bar Lev, Phys. Rev. Lett. 117, 170404 (2016).

[56] H. Tal-Ezer and R. Kosloff, J. Chem. Phys. 81, 3967 (1984).

[57] C. Leforestier, R. Bisseling, C. Cerjan, M. Feit, R. Friesner, A. Guldberg, A. Hammerich, G. Jolicard, W. Karrlein, H.-D. Meyer, N. Lipkin, O. Roncero, and R. Kosloff, J. Comput. Phys. 94, 59 (1991).

[58] H. Fehske and R. Schneider, Computational Many-Particle Physics (Springer, Berlin, 2008).

[59] J. Haegeman, J. I. Cirac, T. J. Osborne, I. Pižorn, H. Verschelde, and F. Verstraete, Phys. Rev. Lett. 107, 070601 (2011).

[60] T. Koffel, M. Lewenstein, and L. Tagliacozzo, Phys. Rev. Lett. 109, 267203 (2012).

[61] J. Haegeman, C. Lubich, I. Oseledets, B. Vandereycken, and F. Verstraete, Phys. Rev. B 94, 165116 (2016).

[62] S. Paeckel, T. Köhler, A. Swoboda, S. R. Manmana, U. Schollwöck, and C. Hubig, Ann. Phys. 411, 167998 (2019).

[63] P. Naldesi, E. Ercolessi, and T. Roscilde, SciPost Phys. 1, 010 (2016).

[64] X. Wei, C. Cheng, G. Xianlong, and R. Mondaini, Phys. Rev. B 99, 165137 (2019).

[65] R. Yao and J. Zakrzewski, Phys. Rev. B 102, 014310 (2020).

[66] Q. Guo, C. Cheng, Z.-H. Sun, Z. Song, H. Li, Z. Wang, W. Ren, H. Dong, D. Zheng, Y.-R. Zhang, R. Mondaini, H. Fan, and H. Wang, arXiv:1912.02818.
[67] T. C. Berkelbach and D. R. Reichman, Phys. Rev. B 81, 224429 (2010).

[68] K. Agarwal, S. Gopalakrishnan, M. Knap, M. Müller, and E. Demler, Phys. Rev. Lett. 114, 160401 (2015).

[69] S. Bera, H. Schomerus, F. Heidrich-Meisner, and J. H. Bardarson, Phys. Rev. Lett. 115, 046603 (2015).

[70] S. Bera, G. De Tomasi, F. Weiner, and F. Evers, Phys. Rev. Lett. 118, 196801 (2017).

[71] L. Herviou, S. Bera, and J. H. Bardarson, Phys. Rev. B 99, 134205 (2019).

[72] L. Colmenarez, P. A. McClarty, M. Haque, and D. J. Luitz, SciPost Phys. 7, 064 (2019).

[73] P. Sierant and J. Zakrzewski, Phys. Rev. B 101, 104201 (2020).

[74] Y. Y. Atas, E. Bogomolny, O. Giraud, and G. Roux, Phys. Rev. Lett. 110, 084101 (2013).

[75] C. Lanczos, J. Res. Natl. Bur. Stand. 45, 255 (1950).

[76] S. R. White, Phys. Rev. Lett. 69, 2863 (1992).

[77] S. R. White, Phys. Rev. B 48, 10345 (1993).

[78] U. Schollwöck, Rev. Mod. Phys. 77, 259 (2005).

[79] U. Schollwöck, Ann. Phys. 326, 96 (2011).

[80] R. Orús, Ann. Phys. 349, 117 (2014).

[81] See Supplemental Material at http://link.aps.org/supplemental/ 10.1103/PhysRevResearch.2.032045 for additional results and brief description of computational methods performed via MPS, which includes Refs. [82,83].

[82] T. Chanda, J. Zakrzewski, M. Lewenstein, and L. Tagliacozzo, Phys. Rev. Lett. 124, 180602 (2020).

[83] S. Goto and I. Danshita, Phys. Rev. B 99, 054307 (2019).

[84] D. J. Luitz, N. Laflorencie, and F. Alet, Phys. Rev. B 93, 060201(R) (2016).

[85] L. D’Alessio, Y. Kafri, A. Polkovnikov, and M. Rigol, Adv. Phys. 65, 239 (2016).

[86] K. Agarwal, E. Altman, E. Demler, S. Gopalakrishnan, D. A. Huse, and M. Knap, Ann. Phys. 529, 1600326 (2017).

[87] H. P. Lüschen, P. Bordia, S. Scherg, F. Alet, E. Altman, U. Schneider, and I. Bloch, Phys. Rev. Lett. 119, 260401 (2017).

[88] F. Weiner, F. Evers, and S. Bera, Phys. Rev. B 100, 104204 (2019).

[89] E. J. Torres-Herrera and L. F. Santos, Phys. Rev. B 92, 014208 (2015).

[90] E. J. Torres-Herrera and L. F. Santos, Philos. Trans. Royal Soc. London, Ser. A 375, 20160434 (2017).

[91] E. J. Torres-Herrera, A. M. García-García, and L. F. Santos, Phys. Rev. B 97, 060303(R) (2018).

[92] M. Schiulaz, E. J. Torres-Herrera, and L. F. Santos, Phys. Rev. B 99, 174313 (2019).

[93] P. Sierant and J. Zakrzewski, New J. Phys. 20, 043032 (2018).

[94] P. Brighi, D. Abanin, and M. Serbyn, arXiv:2005.02999.

[95] https://itensor.org. 